\title{
Anestesia obstétrica en obesa mórbida: Consideraciones
}

\author{
BARBARA M SCAVONE ${ }^{1}$, SERGIO CERDA SM. $^{2}$
}

1 Profesora de Anestesia y Cuidados Críticos, Directora Anestesia Obstetrica, Depto de Anestesia y Cuidados Intensivos, Universidad de Chicago.

2 Profesor Asociado Anestesiología, Depto Obstetricia y Ginecología, Hospital Clínico Universidad de Chile.

\section{I.- Prevalencia}

Utilizando como parámetro el Índice de Masa Corporal (IMC) los datos del Centro de Enfermedades (CDC, Atlanta) indican que un 34\% de los norteamericanos tienen sobrepeso (IMC: $25-$ $\left.29,9 \mathrm{~kg} / \mathrm{m}^{2}\right)$, un $34 \%$ son obesos $\left(\geq 30 \mathrm{~kg} / \mathrm{m}^{2}\right)$ y que un $6 \%$ son obesos extremos $\left(\geq 40 \mathrm{~kg} / \mathrm{m}^{2}\right)$ (Tabla 1). Estas tasas son más altas en la población de mujeres México-Americanas y de Color No Hispánicas ${ }^{1}$. Estas tasas pueden haber llegado actualmente en EEUU a un plateau.

Los datos en Chile no son claros, si bien en las últimas 3 décadas se ha demostrado una eventual erradicación de la desnutrición, la obesidad es un problema álgido de salud pública. Los datos apuntan a un incremento de embarazadas obesas de 13\% en (1987) hasta un 33\% (2000) $)^{2}$.

La definición de obesidad en embarazo no es clara. El IMC se calcula usando el peso previo al embarazo, o, si este peso es desconocido se utiliza el primer peso registrado en control prenatal ${ }^{3}$. En 1993, la Sociedad Americana de Obstetras y Ginecólogos publicó sus clasificaciones (según IMC) de peso materno y óptima ganancia de peso en embarazo (Tabla 2). La ganancia de peso en $\mathrm{kg} / \mathrm{sem}$ es aplicable sólo a $2^{\mathrm{o}}$ y $3^{\text {er }}$ trimestres de embarazo.

\section{II.- Morbimortalidad asociada a obesidad}

La obesidad se asocia a alteraciones anatómicas, fisiológicas y bioquímicas que afectan virtualmente a todos los sistemas.

\section{Respiratorios}

La función respiratoria debe compensar la sobrecarga de masa grasa de tórax y abdomen, esto se traduce en aumento del trabajo respira-

Tabla 1. Índice de Masa Corporal

\begin{tabular}{|cccccc|}
\hline & Magra & Normal & Sobrepeso & Obeso & Obeso extremo \\
\hline $\operatorname{IMC~}\left(\mathrm{kg} / \mathrm{m}^{2}\right)$ & $<18,5$ & $18,5-24,9$ & $25,0-29,9$ & $\geq 30$ & $\geq 40$ \\
\hline
\end{tabular}

Tabla 2. IMC y Ganancia Optima de Peso en Embarazo

\begin{tabular}{|lccccc|}
\hline & Magra & Normal & Sobrepeso & Obeso & Gemelar \\
IMC & $<19,8$ & $19,8-26,0$ & $26,1-29,0$ & $>29$ & \\
Ganancia peso $(\mathrm{kg})$ & $10,9-18,2$ & $11,4-15,9$ & $6,8-11,4$ & 6,8 & $15,9-20,4$ \\
Tasa $(\mathrm{kg} / \mathrm{sem})$ & 570 & 450 & 320 & 230 & 680 \\
\hline
\end{tabular}

Correspondencia a:

scerdasm@gmail.com 
torio y del consumo de $\mathrm{O}_{2}$ y producción de $\mathrm{CO}_{2}$. Hay reducción de los volúmenes y capacidades pulmonares que determinan una Capacidad Residual Funcional dentro de las capacidades de cierre. Es así que existirá cierre de la vía aérea a ventilación con volumen corriente, particularmente en posición supina y Trendelenburg. El cierre de la vía aérea en las zonas dependientes del pulmón desvía la ventilación a zonas no dependientes y no preferentemente perfundidas, generando alteración de la relación ventilación/ perfusión (shunt) e hipoxemia arterial relativa. Eventualmente la reducción de compliance torácica - volúmenes pulmonares y el aumento de la resistencia respiratoria pueden determinar hipertensión pulmonar, hipoxemia y falla biventricu$\operatorname{lar}^{4,5,6}$ (Tabla 3 ).

\section{Cardiovasculares}

Entre los más destacados se haya un deterioro de la vasodilatación inducida con hipertensión resultante, además de hipertrofia auricular y ventricular izquierdas. Se exacerba el aumento del gasto cardíaco a expensas de incremento de la frecuencia cardíaca más que de volumen plasmático. Se puede asociar hipertensión pulmonar proporcional al aumento de gasto cardíaco/volemia.

Se pueden manifestar o agravar enfermedades cardiovasculares coexistentes, aumentan las muertes de causa cardiovascular y la miocardiopatía periparto. La relación entre obesidad e hipertensión es frecuentemente observada, esta se presenta tanto para hipertensión esencial como para trastornos hipertensivos del embarazo. Aún cuando la obesidad determina un aumento al doble del riesgo de hipertensión gestacional y preeclampsia, no se ha encontrado asociada con severidad de Síndrome de HELLP ${ }^{7,8}$. El mecanismo para el aumento del riesgo de trastornos hipertensivos del embarazo en obesidad es desconocido, pero se especula que relaciona con aquellos factores que aumentan riesgo cardiovascular: resistencia insulínica e inflamación subclínica9

\section{Otros efectos}

Los datos son diversos pero la mayoría de los expertos asumen que los individuos obesos muestran un retardo del vaciamiento gástrico y por lo tanto, tienen un riesgo aumentado de aspiración pulmonar de contenido gástrico.

Las pacientes obesas tienen mayor probabilidad de tener diabetes gestacional, por una exagerada insulinoresistencia. La incidencia de Diabetes Gestacional en embarazadas obesas se estima entre 6-8\%. La magnitud del riesgo se correlaciona positivamente con el aumento de peso materno?.

Esta asociación sitúa a estas pacientes en riesgo 2 veces mayor de hipertensión del embarazo, macrosomía fetal y diabetes gestacional ${ }^{10}$.

La obesidad aumenta la frecuencia de enfermedad tromboembólica incluyendo el periparto y el postoperatorio. En estudios retrospectivos se encuentra una incidencia de $2,5 \%$ vs $0,6 \%$ en población obesa y de peso normal respectivamente $^{11,12,13}$.

La obesidad y el Síndrome metabólico están asociados con apnea obstructiva del sueño, entidad que aumenta el riesgo de depresión respi-

Tabla 3. Cambios Fisiopatológicos y Obesidad

\begin{tabular}{|lll|}
\hline \multicolumn{1}{|c|}{ Sistema } & \multicolumn{1}{c|}{ Descripción } & \multicolumn{1}{c|}{ Cambio (Obesidad) } \\
\hline \multirow{3}{*}{ Respiratorio } & Consumo de $\mathrm{O}_{2}$ & Aumentado \\
& Producción ${\mathrm{de} \mathrm{CO}_{2}}$ & Aumentado \\
& Capacidad Residual Funcional & Disminuida \\
& Volumen Reserva Espiratoria & Disminuida \\
& Anormalidad Ventilación/Perfusión & Aumentado \\
\hline \multirow{3}{*}{ Cardiovascular } & Gasto Cardíaco & Aumentado \\
& Volemia & Aumentado \\
\hline Endocrino/metabólico & Hipertensión arterial & Aumentado \\
& Tolerancia a Glucosa & Disminuida \\
& Hipotiroidismo & Aumentado \\
\hline Gastrointestinal & Hernia Hiatal & Aumentado \\
& Presión intraabdominal & Aumentado \\
\hline
\end{tabular}


ratoria en respuesta a opioides y sedantes ${ }^{14}$. Las embarazadas tienen mayor frecuencia de screening positivo para apnea obstructiva del sueño que las no embarazadas, por lo tanto, se postula que el embarazo aumenta su incidencia o exacerba sus manifestaciones ${ }^{15,16}$. Además las embarazadas obesas tienen mayor frecuencia de trastornos respiratorios del sueño que las no-obesas ${ }^{17}$.

\section{III.- Alteraciones en práctica y outcome obs- tétrico en embarazada obesa}

En términos de alteraciones anteparto, la obesidad se ha asociado con un alto riesgo de aborto espontáneo en aquellas pacientes obesas mórbidas que han recibido tratamientos de reproducción asistida (no así para los embarazos espontáneos en obesas) ${ }^{18}$. Así como la relación entre obesidad materna y parto prematura no está bien dilucidada, hay consistente evidencia de que los embarazos de post-término aumentan con la obesidad materna ${ }^{19,20}$.

Las embarazadas obesas tienen aumento en la frecuencia de Inducción de parto, trabajo de parto disfuncional, cesárea por distocia y cesárea de emergencia ${ }^{21,22,23}$.

Los patrones de progresión de Trabajo de Parto en obesas no están bien caracterizados, sin embargo, son conocidas las alteraciones intraparto, que incluyen un aumento de inducciones (RR 1,7 - 2,2 veces mayor) y una asociación significativa entre obesidad y operación Cesárea, que es independiente de los factores antenatales asociadas a la obesidad, ej baja talla materna, alto peso del RN, edad gestacional ${ }^{24,25,26}$.

La obesidad aumenta el riesgo de macrosomía, de RN grandes para la edad gestacional y de retención de hombros ${ }^{24,27,28}$. Los $\mathrm{RN}$ macrosómicos nacidos de madres obesas tienen además un riesgo aumentado de obesidad en la infancia y vida adulta ${ }^{28,29}$.

Como se ha mencionado antes, las pacientes obesas tienen un riesgo más bajo de parto prematuro y de RN de bajo peso, y un riesgo mayor de parto de post-término ${ }^{28}$. Finalmente la obesidad confiere un riesgo mayor de muerte fetal in útero, muerte neonatal precoz y aumento de mortalidad perinatal ${ }^{30}$, además de aumento del riesgo de malformaciones congénitas, especialmente defectos del tubo neural ${ }^{3}$.
La combinación de obesidad y preeclampsia se asocia con mayor frecuencia de mortalidad temprana de las madres ${ }^{31}$. En los resultados perioperatorios, estas pacientes presentan aumento del riesgo de infección, dehiscencia de herida y tromboembolismo ${ }^{32}$.

\section{Implicancias anestésicas}

Debe contarse con visita pre-anestésica precoz para todas las embarazadas obesas, y se debe generar un plan de trabajo multidisciplinario, con participación de obstetras, anestesiólogos, neonatologos e intensivistas. Específicamente, para las pacientes con apnea obstructiva del sueño, ASA recomienda evaluación y plan de atención definido anticipadamente ${ }^{33}$.

Las alteraciones anatómicas en la embarazada obesa inducen dificultades para determinar referencia anatómicas para elección del sitio de punción neuraxial, líneas arteriales, y alteraciones del acceso a la vía aérea. Agujas epidurales y espinales de mayor longitud deben estar disponibles. Con estas agujas, el operador debe estar consciente de que pequeños cambios del ángulo de aproximación en el sitio de punción, se traducen en desviaciones significativas en la trayectoria de la aguja hacia su objetivo neuraxial.

Específicamente en la técnica epidural continua, una vez que el espacio epidural es identificado y el catéter es introducido, su fijación definitiva debe realizarse con el paciente en decúbito lateral y con las extremidades inferiores en posición de extensión, para evitar así la tracción que ejercerían los tejidos lumbares, y que tiende a sacar el catéter desde la posición epidural.

Ante dificultades con la técnica epidural, una combinada espinal -epidural o una espinal continua ofrecen ventajas comparativas. En ambas, la confirmación definitiva de LCR y la inserción de un catéter, que permita la extensión de la analgesia o su conversión a anestesia quirúrgica, además de analgesia postoperatoria son de la mayor utilidad en el manejo anestésico de pacientes obesas ${ }^{35,36}$.

Se ha descrito una mayor frecuencia de hipotensión y alteraciones de la Frecuencia Cardíaca Fetal después de analgesia neuraxial para Trabajo de Parto en pacientes obesas, además hay mayor frecuencia de falla epidural ${ }^{34,37,38}$.

En Operación Cesárea, la anestesia general es requerida con una mayor frecuencia que en población no obesa ${ }^{34,38}$ con dificultades concomi- 
tantes en el manejo de la vía aérea. Los riesgos de falla de ventilación-falla de intubación y aspiración de contenido gástrico están exacerbados por la obesidad. Como factor relevante, la obesidad es un factor contribuyente de mortalidad materna relacionada a anestesia, especialmente en el período post-operatorio ${ }^{39,40}$.

\section{Analgesia en Trabajo de Parto}

Las pacientes obesas en Trabajo de Parto deben ser evaluadas precozmente después de su admisión al área de Pre-Partos y una analgesia neuraxial precoz debe formar parte del protocolo de manejo.

La posición sentada, que reduce la distancia piel-epidural, y el uso de ultrasonido pueden facilitar el proceso de punción neuraxial.

Las pacientes obesas tienen menores requerimientos de anestésicos, locales epidurales e intratecales , para el establecimiento de un bloqueo, posiblemente debido a menor volumen lumbosacro de $\mathrm{LCR}^{41,42,43,44}$.

La falla de extender un bloqueo epidural analgésico a una anestesia epidural quirúrgica (Cesárea) se puede predecir por el número de top ups necesarios para establecer, o por falla en obtener analgesia efectiva. Una política activa de evaluación continua, en las pacientes obesas, de la funcionalidad del catéter puede evitar la falla epidural para Cesárea ${ }^{45,46}$.

Como se mencionó antes, una consideración especial debe tenerse con el uso de anestesia espinal continua, como una forma de prevenir la falla epidural. La posición intratecal del catéter asegura y facilita la titulación del bloqueo en oposición a un catéter epidural, en pacientes obesas. Un artículo clásico describe un efecto protector de la obesidad en la incidencia de cefalea post punción dural ${ }^{47}$.

\section{Operación Cesárea}

Se debe preferir la anestesia neuraxial, para prevenir las dificultades relativas a ventilación e intubación, está especialmente recomendada en las pacientes obesas con Apnea Obstructiva del Sueño ${ }^{48}$.

Se debe prevenir la posición supina debido a los riesgos de edema pulmonar y muerte súbita de origen cardíaco ${ }^{49}$.

Se debe preferir una técnica continua, debido a que los tiempos operatorios son mayores en po- blación obesa ${ }^{50}$. Muchas de las recomendaciones enumeradas para analgesia en parto vaginal, como dificultades técnicas, y disminución de dosis de anestésicos locales, aplican también para manejo de Cesárea con anestesia neuraxial. Al igual que en Trabajo de Parto, se debe tener en consideración el uso de anestesia espinal continua.

En caso de anestesia general, la laringoscopía otorga una mejor visión con la paciente obesa en posición de rampa ${ }^{51}$. La posición de Fowler se asocia a una pre-oxigenación más eficiente ${ }^{52} \mathrm{y}$ mejor oxigenación postoperatoria ${ }^{53}$.

$\mathrm{Si}$ se require anestesia general se deben seguir las recomendaciones de Manejo de Vía Aérea Difícil en Embarazadas ${ }^{56}$, especial opción debe ser la intubación traqueal vigil con fibrobroncoscopio. Aunque existe probablemente un aumento de los casos: No puedo intubar-No puedo ventilar, es dificil precisar la incidencia de esta situación basado en IMC específicos ${ }^{50}$.

Se debe esperar que estas pacientes tengan una disminución de la reserva de $\mathrm{O}^{2}$ y un rápido tiempo de desaturación arterial durante apnea, asociado a las alteraciones respiratorias inducidas por embarazo y agravadas por la obesidad, como son la disminución de Capacidad Residual Funcional y el aumento de consumo de $\mathrm{O}_{2}$. La Pre-oxigenación es more efficient in the head up position $^{54}$. En el post-operatorio las pacientes obesas tienen un mayor riesgo de obstrucción de la vía aérea y mortalidad ${ }^{40}$.

El anestesista se enfrenta con objetivos contrapuestos, para proveer buena analgesia y aumentar la movilidad de la paciente para reducir la incidencia de tromboembolismo y al mismo tiempo minimizar los requerimientos de opioides. La terapia analgésica multimodal está recomendada, especialmente en antecedentes de apnea obstructiva del sueño.

Se debe proveer analgesia epidural o bloqueo TAP que disminuirán los requerimientos de opioides, en aquellos pacientes que no recibieron morfina intratecal ${ }^{55}$.

Las Guías de la ASA para pacientes con apnea obstructiva del sueño recomiendan oxígeno suplementario con presión positiva continua (CPAP) en pacientes con diagnóstico documentado o en alto riesgo de Apnea Obstructiva del sueño, además de oximetría continua y manejo en una sala de cuidado intensivo en el período postoperatorio precoz. 


\section{Consideraciones farmacológicas}

El embarazo, combinado con la obesidad, determinan alteraciones en la farmacocinética y farmacodinamia de las drogas anestésicas. Ambas, embarazo y obesidad aumentan en forma significativa el volumen de distribución de la mayoría de las drogas utilizadas. Las duración de acción de las drogas altamente lipofílicas (propofol, benzodiazepinas, thiopental) sigue a este aumento del volumen de distribución; mientras que el aumento de la distribución, típicamente reduce el contenido de droga activa, el aumento de triglicéridos, colesterol y ácidos grasos libres observado en la obesidad puede inhibir la unión a proteínas aumentando la fracción libre de la droga activa ${ }^{57}$. En el caso de propofol, su amplio volumen de distribución, es contrarrestado por su rápida vida media de eliminación, determinando, en general una duración similar en pacientes de peso normal y obesas. Las benzodiazepinas y el thiopental tienen largas vida media de eliminación. Fentanilo, sufentanilo y remifentanilo son altamente lipofílicos lo cual debería resultar en un gran volumen de distribución y lenta vida media de eliminación. Es interesante hacer notar que el volumen de distribución de remifentanilo en obesas no embarazadas es menor de lo esperado por su lipofilicidad, además el clearance en esta misma población no es significativamente mas lento que en no obesos. Más aún, el clearance de remifentanilo aumenta significativamente en el embarazo ${ }^{58,59,60}$.

Sevoflurano y desflurano son mucho menos liposolubles que los antiguos agentes halogenados, por lo que proveen un más rápido y consistente perfil de recuperación. A partir de esta característica, tiempos similares de despertar se han descrito en sujetos de peso normal y en obesos para procedimientos entre $2-4 \mathrm{~h}$ de duración. Con exposiciones de corta duración, la biotransformación de los anestésicos volátiles a compuestos fluorados inorgánicos, es limitada en pacientes obesos, lo que resulta un factor protector de hepato y nefrotoxicidad ${ }^{61}$.

Los relajantes musculares, agentes polareshidrofílicos, deberían presentar alteraciones menores en pacientes obesos. En el embarazo se ha descrito una sensibilidad facilitada a los relajantes de tipo aminoesteroidal, situación que no es explicable por farmacocinética ${ }^{62}$. En el caso específico de rocuronio, la duración de acción está significativamente aumentada en pacientes obesos mórbidos si se dosifica según el peso real, por lo que se recomienda usar el peso ideal para la práctica clínica ${ }^{63}$. El uso mandatorio de sugammadex, especialmente en condiciones de No puedo intubar - No puedo ventilar se ha recomendado en el manejo de vía aérea en embarazadas $^{60}$, es así que en obesos mórbidos se recomienda una dosis de $4 \mathrm{mg} / \mathrm{kg}$ para reversión completa de bloqueo neuromuscular profundo ${ }^{64}$. En el caso de succinilcolina, las potencias son comparables en sujetos de peso normal y obesos, los efectos contrapuestos en la actividad de pseudocolinoesterasa, aumento por obesidad y disminución por embarazo, se recomienda el uso de dosis ajustada al peso real para intubación ${ }^{65,66,67}$.

\section{Referencias}

1. Flegal KM, Carroll MD, Ogden CL, Curtin LR. Prevalence and trends in obesity among US adults, 1999-2008. JAMA 2010 Jan;303(3):235-41.

2. Albala C, Vio F, Kain J, Uauy R. Nutrition transition in Latin America: the case of Chile. Nutr Rev 2001 Jun;59(6):170-6.

3. Andreasen KR, Andersen ML, Schantz AL. Obesity and pregnancy. Acta Obstet Gynecol
Scand 2004 Nov;83(11):1022-9.

4. Ladosky W, Botelho MA, Albuquerque JP Jr. Chest mechanics in morbidly obese non-hypoventilated patients. Respir Med 2001 Apr;95(4):281-6.

5. Bananian S, Lehrman SG, Maguire GP. Cardiovascular consequences of sleep-related breathing disorders. Heart Dis 2002 Sep-Oct;4(5):296-305.

6. Tanaka M, Watanabe S, Nishikawa T. Anesthetic management in obese parturients. J
Anesth 1999 Oct;13(4):217-29.

7. Sibai BM, Gordon T, Thom

E, Caritis SN, Klebanoff M, McNellis D et al.; The National Institute of Child Health and $\mathrm{Hu}-$ man Development Network of Maternal-Fetal Medicine Units. Risk factors for preeclampsia in healthy nulliparous women: a prospective multicenter study. Am J Obstet Gynecol 1995 Feb;172(2 Pt 1):642-8.

8. Sibai BM, Ewell M, Levine RJ, Klebanoff MA, Esterlitz J, Cata- 
lano PM et al.; The Calcium for Preeclampsia Prevention (CPEP) Study Group. Risk factors associated with preeclampsia in healthy nulliparous women. Am J Obstet Gynecol 1997 Nov;177(5):1003-10.

9. Nuthalapaty FS, Rouse DJ. The impact of obesity on obstetrical practice and outcome. Clin Obstet Gynecol 2004 Dec;47(4):898913.

10. Metzger BE, Coustan DR; The Organizing Committee. Summary and recommendations of the Fourth International Workshop-Conference on Gestational Diabetes Mellitus. Diabetes Care 1998 Aug;21 Suppl 2:B161-7.

11. Edwards LE, Hellerstedt WL, Alton IR, Story M, Himes JH. Pregnancy complications and birth outcomes in obese and normal-weight women: effects of gestational weight change. Obstet Gynecol 1996 Mar;87(3):38994.

12. Larsen TB, Sørensen HT, Gislum M, Johnsen SP. Maternal smoking, obesity, and risk of venous thromboembolism during pregnancy and the puerperium: a population-based nested case-control study. Thromb Res 2007;120(4):505-9.

13. Rocha AT, de Vasconcellos AG, da Luz Neto ER, Araújo DM, Alves ES, Lopes AA. Risk of venous thromboembolism and efficacy of thromboprophylaxis in hospitalized obese medical patients and in obese patients undergoing bariatric surgery. Obes Surg 2006 Dec;16(12):1645-55.

14. Parish JM, Adam T, Facchiano

L. Relationship of metabolic syndrome and obstructive sleep apnea. J Clin Sleep Med 2007 Aug;3(5):467-72.

15. Roush SF, Bell L. Obstructive sleep apnea in pregnancy. $\mathrm{J}$
Am Board Fam Pract 2004 JulAug;17(4):292-4.

16. Higgins N, Leong E, Park CS, Facco FL, McCarthy RJ, Wong CA. The Berlin Questionnaire for assessment of sleep disordered breathing risk in parturients and non-pregnant women. Int J Obstet Anesth 2011 Jan;20(1):22-5.

17. Maasilta P, Bachour A, Teramo K, Polo O, Laitinen LA. Sleeprelated disordered breathing during pregnancy in obese women. Chest 2001 Nov;120(5):1448-54.

18. Nuthalapaty FS, Rouse DJ. The impact of obesity on obstetrical practice and outcome. Clin Obstet Gynecol 2004 Dec;47(4):898913.

19. Bellver J, Rossal LP, Bosch E, Zúñiga A, Corona JT, Meléndez $\mathrm{F}$ et al. Obesity and the risk of spontaneous abortion after oocyte donation. Fertil Steril 2003 May;79(5):1136-40.

20. Ehrenberg HM, Dierker L, Milluzzi C, Mercer BM. Prevalence of maternal obesity in an urban center. Am J Obstet Gynecol 2002 Nov;187(5):1189-93.

21. Kominiarek MA, Zhang J, Vanveldhuisen P, Troendle J, Beaver J, Hibbard JU. Contemporary labor patterns: the impact of maternal body mass index. Am J Obstet Gynecol 2011 Sep;205(3):244.e1-8.

22. Kominiarek MA, Vanveldhuisen P, Hibbard J, Landy H, Haberman S, Learman L et al.; Consortium on Safe Labor. The maternal body mass index: a strong association with delivery route. Am J Obstet Gynecol 2010 Sep;203(3):264.e1-7.

23. Ekblad U, Grenman S. Maternal weight, weight gain during pregnancy and pregnancy outcome. Int J Gynaecol Obstet 1992 Dec;39(4):277-83.
24. Weiss JL, Malone FD, Emig D, Ball RH, Nyberg DA, Comstock $\mathrm{CH}$ et al.; FASTER Research Consortium. Obesity, obstetric complications and cesarean delivery rate - a population-based screening study. Am J Obstet Gynecol 2004 Apr;190(4):10917.

25. Durnwald CP, Ehrenberg HM, Mercer BM. The impact of maternal obesity and weight gain on vaginal birth after cesarean section success. Am J Obstet Gynecol 2004 Sep;191(3):954-7.

26. Hibbard JU, Gilbert S, Landon MB, Hauth JC, Leveno KJ, Spong CY et al.; National Institute of Child Health and Human Development Maternal-Fetal Medicine Units Network. Trial of labor or repeat cesarean delivery in women with morbid obesity and previous cesarean delivery. Obstet Gynecol 2006 Jul;108(1):125-33.

27. Cedergren MI. Maternal morbid obesity and the risk of adverse pregnancy outcome. Obstet Gynecol 2004 Feb;103(2):219-24.

28. Sebire NJ, Jolly M, Harris JP, Wadsworth J, Joffe M, Beard RW et al. Maternal obesity and pregnancy outcome: a study of 287,213 pregnancies in London. Int J Obes Relat Metab Disord 2001 Aug;25(8):1175-82.

29. Reece EA. Perspectives on obesity, pregnancy and birth outcomes in the United States: the scope of the problem. Am J Obstet Gynecol 2008 Jan;198(1):237.

30. Nohr EA, Bech BH, Davies MJ, Frydenberg M, Henriksen TB, Olsen J. Prepregnancy obesity and fetal death: a study within the Danish National Birth Cohort. Obstet Gynecol 2005 Aug;106(2):250-9.

31. Samuels-Kalow ME, Funai EF, 
Buhimschi C, et al. Prepregnancy body mass index, hypertensive disorders of pregnancy, and long-term maternal mortality. Am J Obstet Gynecol 2007; 197: 490.e1-490.e627,28. 10.1016/j. ajog.2007.04.043.

32. Catalano PM. Management of obesity in pregnancy. Obstet Gynecol 2007 Feb;109(2 Pt 1):419-33.

33. Gross JB, Bachenberg KL, Benumof JL, Caplan RA, Connis RT, Coté CJ et al.; American Society of Anesthesiologists Task Force on Perioperative Management. Practice guidelines for the perioperative management of patients with obstructive sleep apnea: a report by the American Society of Anesthesiologists Task Force on Perioperative Management of patients with obstructive sleep apnea. Anesthesiology 2006 May;104(5):1081-93.

34. Hood DD, Dewan DM. Anesthetic and obstetric outcome in morbidly obese parturients. Anesthesiology 1993 Dec;79(6):1210-8.

35. Kuczkowski KM. Labor analgesia for the morbidly obese parturient: an old problem-new solution. Arch Gynecol Obstet 2005 Apr;271(4):302-3.

36. Michaloudis D, Fraidakis O, Petrou A, Farmakalidou H, Neonaki M, Christodoulakis M et al. Continuous spinal anesthesia/analgesia for perioperative management of morbidly obese patients undergoing laparotomy for gastroplastic surgery. Obes Surg 2000 Jun;10(3):220-9.

37. Vricella LK, Louis JM, Mercer $\mathrm{BM}$, et al. Impact of morbid obesity on epidural anesthesia complications in labor. Am J Obstet Gynecol 2011; 205: 370. e1-6. 10.1016/j.ajog.2011.06.085.

38. Vricella LK, Louis JM, Mercer BM, Bolden N. Anesthesia com- plications during scheduled cesarean delivery for morbidly obese women. Am J Obstet Gynecol 2010 Sep;203(3):276.e1-5.

39. Endler GC, Mariona FG, Sokol RJ, Stevenson LB. Anesthesiarelated maternal mortality in Michigan, 1972 to 1984. Am J Obstet Gynecol 1988 Jul;159(1):187-93.

40. Mhyre JM, Riesner MN, Polley LS, Naughton NN. A series of anesthesia-related maternal deaths in Michigan, 19852003. Anesthesiology 2007 Jun;106(6):1096-104.

41. Panni MK, Columb MO. Obese parturients have lower epidural local anaesthetic requirements for analgesia in labour. $\mathrm{Br} \mathrm{J}$ Anaesth 2006 Jan;96(1):106-10.

42. Hodgkinson R, Husain FJ. Obesity, gravity, and spread of epidural anesthesia. Anesth Analg 1981 Jun;60(6):421-4.

43. McCulloch WJ, Littlewood DG. Influence of obesity on spinal analgesia with isobaric $0.5 \%$ bupivacaine. Br J Anaesth 1986 Jun;58(6):610-4.

44. Sullivan JT, Grouper S, Walker MT, Parrish TB, McCarthy RJ, Wong CA. Lumbosacral cerebrospinal fluid volume in humans using three-dimensional magnetic resonance imaging. Anesth Analg 2006 Nov;103(5):1306-10.

45. Eappen S, Blinn A, Segal S. Incidence of epidural catheter replacement in parturients: a retrospective chart review. Int J Obstet Anesth 1998 Oct;7(4):220-5.

46. Riley ET, Papasin J. Epidural catheter function during labor predicts anesthetic efficacy for subsequent cesarean delivery. Int J Obstet Anesth 2002 Apr;11(2):81-4.

47. Faure E, Moreno R, Thisted R. Incidence of postdural puncture headache in morbidly obese parturients. Reg Anesth 1994 Sep-Oct;19(5):361-3.

48. Gross JB, Bachenberg KL, Benumof JL, Caplan RA, Connis RT, Coté CJ et al.; American Society of Anesthesiologists Task Force on Perioperative Management. Practice guidelines for the perioperative management of patients with obstructive sleep apnea: a report by the American Society of Anesthesiologists Task Force on Perioperative Management of patients with obstructive sleep apnea. Anesthesiology 2006 May;104(5):1081-93.

49. Tsueda K, Debrand M, Zeok SS, Wright BD, Griffin WO. Obesity supine death syndrome: reports of two morbidly obese patients. Anesth Analg 1979 JulAug;58(4):345-7.

50. Perlow JH, Morgan MA. Massive maternal obesity and perioperative cesarean morbidity. Am J Obstet Gynecol 1994 Feb;170(2):560-5.

51. Collins JS, Lemmens HJ, Brodsky JB, Brock-Utne JG, Levitan RM. Laryngoscopy and morbid obesity: a comparison of the "sniff" and "ramped" positions. Obes Surg 2004 Oct;14(9):11715.

52. Brodsky JB, Lemmens HJ, Brock-Utne JG, Vierra M, Saidman LJ. Morbid obesity and tracheal intubation. Anesth Analg 2002 Mar;94(3):732-6.

53. Vaughan RW, Bauer S, Wise L. Effect of position (semirecumbent versus supine) on postoperative oxygenation in markedly obese subjects. Anesth Analg 1976 Jan-Feb;55(1):37-41.

54. Dixon BJ, Dixon JB, Carden JR et al. Preoxygenation is more effective in the $25^{\circ}$ head-up position than in the supine position in severely obese patients. 
Anesthesiology 2005;102:11105.

55. McDonnell JG, Curley G, Carney J, Benton A, Costello J, Maharaj $\mathrm{CH}$ et al. The analgesic efficacy of transversus abdominis plane block after cesarean delivery: a randomized controlled trial. Anesth Analg 2008 Jan;106(1):186-91.

56.

57. Casati A, Putzu M. Anesthesia in the obese patient: pharmacokinetic considerations. J Clin Anesth 2005 Mar; 17(2):134-45.

58. Egan TD, Huizinga B, Gupta SK, Jaarsma RL, Sperry RJ, Yee JB et al. Remifentanil pharmacokinetics in obese versus lean patients. Anesthesiology 1998 Sep;89(3):562-73.

59. Kan RE, Hughes SC, Rosen MA, Kessin C, Preston PG, Lobo EP. Intravenous remifentanil: placental transfer, maternal and neonatal effects. Anesthesiology 1998 Jun;88(6):1467-74.

60.

61. Frink EJ Jr, Malan TP Jr, Brown EA, Morgan S, Brown BR Jr. Plasma inorganic fluoride levels with sevoflurane anesthesia in morbidly obese and nonobese patients. Anesth Analg 1993 Jun;76(6):1333-7.

62. Guay J, Grenier Y, Varin F. Clinical pharmacokinetics of neuromuscular relaxants in pregnancy. Clin Pharmacokinet 1998 Jun;34(6):483-96.

63. Leykin Y, Pellis T, Lucca M, Lomangino G, Marzano B, Gullo A. The pharmacodynamic effects of rocuronium when dosed according to real body weight or ideal body weight in morbidly obese patients. Anesth Analg 2004 Oct;99(4):1086-9.
64. Loupec T, Frasca D, Rousseau N, Faure JP, Mimoz O, Debaene B. Appropriate dosing of sugammadex to reverse deep rocuronium-induced neuromuscular blockade in morbidly obese patients. Anaesthesia 2016 Mar;71(3):265-72.

65. Rose JB, Theroux MC, Katz MS. The potency of succinylcholine in obese adolescents. Anesth Analg 2000 Mar;90(3):576-8.

66. Bentley JB, Borel JD, Vaughan RW, Gandolfi AJ. Weight, pseudocholinesterase activity, and succinylcholine requirement. Anesthesiology 1982 Jul;57(1):48-9.

67. Evans RT, Wroe JM. Plasma cholinesterase changes during pregnancy. Their interpretation as a cause of suxamethoniuminduced apnoea. Anaesthesia $1980 \mathrm{Jul} ; 35(7): 651-4$. 